\title{
UNSTEADY, LAMINAR FLOW SIMULATIONS USING THE NONLINEAR DISTURBANCE EQUATIONS
}

\author{
R. P. Hansen ${ }^{*}$ L. N. Long ${ }^{\dagger}$ and P. J. Morris \\ Department of Aerospde Engineering \\ The Pennsylvania State University \\ University Park, PA 16802
}

\begin{abstract}
The results of numerical simulations using the Nonlinear Disturbance Equations (NLDE) for viscous test cases in laminar flow are presented. The NLDE are implemented in a two-dimensional, finite volume code to test the algorithm's properties for viscous, wall-bounded flows. Testing focused on the accuracy of unsteady phenomena. An overview of the method, details on the implementation of boundary conditions, and results for a benchmark scattering problem and viscous flow over a circular cylinder are presented. The results show this method converges to a time accurate periodic condition using a variety of inputs for the mean flow field, and under some circumstances may converge substantially faster to the periodic state compared to the same code using a standard Navier-Stokes formulation.
\end{abstract}

\section{Introduction}

This paper describes recent developments in the use of the Nonlinear Disturbance Equations (NLDE) for the numerical prediction of unsteady flows. This work extends the applicability of the NLDE beyond the previous works by Morris et al. ${ }^{1,2,3}$, Liu et $a l^{4}{ }^{4}$, and Liu and Long ${ }^{5}$. These earlier works used the NLDE for either an inviscid wall-bounded flow, or free shear flows. In this paper, the NLDE method is applied to wallbounded, viscous flows. Results for unsteady inviscid and viscous, laminar test cases are reported here.

Previous success using the NLDE involved the simulation of jets and inviscid wall-bounded flows. Morris et al. ${ }^{1}$ implemented the NLDE in the solution of jet noise. In this work the disturbances were assumed inviscid. A finite difference method on a structured grid was used and the code was implemented on parallel hardware. This work was followed by further research considering both circular and rectangular jets, as well as incorporating a sub-grid scale model ${ }^{2}$. Comparisons with experimental data showed the method to accurately predict radiated noise levels to within $5 \mathrm{~dB}$. More recent calculations ${ }^{6}$ have improved the absolute accuracy of the predictions. In a low-speed application, the airwakes behind ships were studied using the $\mathrm{NLDE}^{4}$. This simulation was also conducted on a structured grid for inviscid flow, and successfully predicted the wake structure behind a landing helicopter aide (LHA) and a generic frigate for various wind conditions. Application of the NLDE to the aerodynamics and aeroacoustics of multi-hole engine inlet liners ${ }^{5}$ also demonstrated the versatility of this formulation. The most current efforts using NLDE include additional jet noise studies by Scheidegger $e t$ al. ${ }^{6}$, use of large-eddy simulation with NLDE for wall-bounded flows by Chyczewski et al. ${ }^{7}$, and a non-conservative NLDE formulation by Long ${ }^{8}$. Sezer-Uzol and Long ${ }^{9}$ have implemented a splitting method related to NLDE for calculating the vortex structures in wakes.

Complete details of the NLDE are given by Morris et $a l .{ }^{1}$. An overview is given here. The method is based on the traditional splitting of the flow variables into a mean component and a perturbation about that mean. The mean component may be obtained from any traditional CFD code. In the case of the laminar tests conducted here, the Navier-Stokes equations are solved using the standard Jameson, Schmidt, and Turkel finite volume formulation ${ }^{10}$ to provide the mean. When turbulence is involved, a code providing the Reynolds (time) averaged results can be used. The mean flow solution is then input into the NLDE equation set to solve directly for the perturbations. The NLDE solver used here is a modified version of the same Jameson style finite volume code used to obtain the mean. Of particular interest in this paper is the comparison of the NLDE solutions for different types of mean flow input.

There are several other advantages of this decomposition method. The magnitude of the perturbations sought that yield aeroacoustic information are often several orders of magnitude smaller than the mean quantities. By solving for the perturbations in a separate step, the numerical round-off errors affecting

\footnotetext{
* Doctoral Candidate, LTC, United States Army

${ }^{\dagger}$ Professor, Associate Fellow, AIAA.

¥ Boeing / A.D. Welliver Professor, Associate Fellow, AIAA

Copyright (C) 2000 by Hansen, Long, and Morris. Published by the American Institute of Aeronautics and Astronautics, Inc. with permission
} 
the acoustic solution are reduced. When the mean flow is obtained as described above, it automatically satisfies all wall and farfield boundary conditions. This allows the boundaries during the NLDE solution to be treated using highly effective radiation and outflow conditions developed for Computational Aeroacoustic (CAA) applications. The contamination of aeroacoustic information from reflections at the computational boundaries is minimized as a result. The NLDE approach also allows the use of efficient implicit or multigrid CFD schemes for the mean flow, and a higherorder CAA algorithm for the unsteady flow.

\section{Governing Equations}

The NLDE equations are obtained from the full, time-dependent, Navier-Stokes equations. In two dimensions they may be written in the form:

$$
\frac{\partial q}{\partial t}+\frac{\partial F}{\partial x}+\frac{\partial G}{\partial y}=\frac{\partial R}{\partial x}+\frac{\partial S}{\partial y}
$$

The conserved vector variable is given by:

$$
q=\left\{\begin{array}{c}
\rho \\
\rho u \\
\rho v \\
e
\end{array}\right\}
$$

where $\rho$ is the fluid density, $u$ and $v$ are the velocity components, and $e$ is the total energy per unit volume of fluid defined as

$$
e=\frac{p}{\gamma-1}+\frac{1}{2} \rho\left(u^{2}+v^{2}\right)
$$

where $p$ and $\gamma$ are the pressure and specific heat ratio, respectively. Equation 3 assumes the ideal gas law is valid. The inviscid fluxes, $F$ and $G$, and the viscous fluxes, $R$ and $S$, are the same conserved variables defined by Morris et al. ${ }^{1}$

Each variable (density, velocity components, energy, and pressure) is decomposed into a mean and a perturbation and substituted into equation 1 . The resulting set of equations are the same as a conventional Reynolds decomposition, except they are not time averaged. The terms involving purely mean flow, obtained from Reynolds averaging, are instead placed on the right-hand side of the equations. All terms containing unsteady components are placed on the lefthand side of the equations. In this form they are called the Nonlinear Disturbance Equations (NLDE) and take the form (in two dimensions):

$$
\begin{aligned}
& \frac{\partial q^{\prime}}{\partial t}+\frac{\partial\left(F^{\prime}+F_{n}{ }^{\prime}\right)}{\partial x}+\frac{\partial\left(G^{\prime}+G_{n}{ }^{\prime}\right)}{\partial y} \\
&-\frac{\partial\left(R^{\prime}+R_{n}{ }^{\prime}\right)}{\partial x}-\frac{\partial\left(S^{\prime}+S_{n}{ }^{\prime}\right)}{\partial y}=Q_{o}
\end{aligned}
$$

The terms under the time derivative and first spatial derivative may be written as:

$$
q^{\prime}=\left\{\begin{array}{c}
\rho^{\prime} \\
\rho_{o} u^{\prime}+\rho^{\prime} u_{o}+\rho^{\prime} u^{\prime} \\
\rho_{o} v^{\prime}+\rho^{\prime} v_{o}+\rho^{\prime} v^{\prime} \\
e^{\prime}
\end{array}\right\}
$$

$$
F^{\prime}=\left\{\begin{array}{c}
\rho_{o} u^{\prime}+\rho^{\prime} u_{o} \\
\rho_{o} u^{\prime}+\rho^{\prime} u_{o}^{2}+2 \rho_{o} u_{o} u^{\prime}+p^{\prime} \\
\rho_{o} u_{o} v^{\prime}+\rho_{o} v_{o} u^{\prime}+\rho^{\prime} u_{o} v_{o} \\
e^{\prime}\left(u_{o}+p_{o}\right)+u_{o}\left(e^{\prime}+p^{\prime}\right)
\end{array}\right\}
$$

$$
F_{n}^{\prime}=\left\{\begin{array}{c}
\rho^{\prime} u^{\prime} \\
\rho_{o} u^{\prime 2}+\rho^{\prime} u^{\prime 2}+2 \rho^{\prime} u^{\prime} u_{o} u^{\prime} \\
\rho_{o} u^{\prime} v^{\prime}+\rho^{\prime} v^{\prime} u_{o}+\rho^{\prime} u^{\prime} v_{o}+\rho^{\prime} u^{\prime} v^{\prime} \\
u^{\prime}\left(e^{\prime}+p^{\prime}\right)
\end{array}\right\}
$$

The right-hand side of equation 4 consists of:

$$
Q_{o}=-\left(\frac{\partial q_{o}}{\partial t}+\frac{\partial F_{o}}{\partial x}+\frac{\partial G_{o}}{\partial y}\right)+\frac{\partial R_{o}}{\partial x}+\frac{\partial S_{o}}{\partial y}
$$

The flux terms associated with $G, R$, and $S$ on the lefthand side can be similarly expanded. In this form, $q^{\prime}$ becomes a new vector of conserved variables, $F^{\prime}, G^{\prime}, R^{\prime}$, and $S^{\prime}$ contain linear perturbation terms, $F_{n}{ }^{\prime}, G_{n}{ }^{\prime}, R_{n}{ }^{\prime}$, and $S_{n}{ }^{\prime}$ contain nonlinear terms.

The right-hand side terms defined in $Q_{o}$ (equation 8) are the same as the Navier-Stokes terms with the mean (subscript "o") variable substituted for the total variable. Previous papers on the NLDE assumed the time derivative of the mean quantities $\left(\partial \mathrm{q}_{\mathrm{o}} / \partial \mathrm{t}\right)$ was zero. These earlier references defined $Q_{o}$ as

$$
Q_{o}=-\left(\frac{\partial F_{o}}{\partial x}+\frac{\partial G_{o}}{\partial y}\right)+\frac{\partial R_{o}}{\partial x}+\frac{\partial S_{o}}{\partial y}
$$

The current effort retains the time derivative of the mean quantities $\left(\partial q_{o} / \partial t\right)$ in the definition of $Q_{o}$ in an attempt to broaden the range of mean flows that may be studied as inputs to the NLDE formulation. Specifically, this admitted the possibility of using a "snapshot" of a time accurate, instantaneous, flowfield as an NLDE mean 


\begin{tabular}{|c|c|c|c|c|}
\hline Mean Input Type & $\frac{\partial q_{o}}{\partial t}$ & $\begin{array}{l}\text { Sum } \\
\text { of } \\
\text { Fluxes }\end{array}$ & $\mathrm{Q}_{\mathrm{o}}$ & Notes \\
\hline $\begin{array}{l}\text { Converged, Steady } \\
\frac{\partial F_{o}}{\partial x}+\frac{\partial G_{o}}{\partial y}=\frac{\partial R_{o}}{\partial x}+\frac{\partial S_{o}}{\partial y}\end{array}$ & 0 & 0 & 0 & \\
\hline $\begin{array}{l}\text { Time-Averaged } \\
\vec{q}_{o}=\frac{1}{N} \sum_{i=1}^{N} \vec{q}_{i}\end{array}$ & 0 & $\neq 0$ & $\neq 0$ & \\
\hline $\begin{array}{l}\text { Freestream } \\
\qquad \vec{q}_{o}=\vec{q}_{\infty}\end{array}$ & 0 & 0 & 0 & $\begin{array}{l}\text { no mean flow } \\
\text { solution } \\
\text { required }\end{array}$ \\
\hline $\begin{array}{l}\text { Instantaneous } \\
\vec{q}_{o}=\vec{q}(\vec{x}, t)\end{array}$ & $\neq 0$ & $\neq 0$ & 0 & $\begin{array}{l}\text { has not } \\
\text { produced } \\
\text { correct } \\
\text { solutions }\end{array}$ \\
\hline Converged, RANS & 0 & $\neq 0$ & $\neq 0$ & $\begin{array}{c}\mathrm{Q}_{\mathrm{o}} \text { contains } \\
\text { Reynolds } \\
\text { stresses }\end{array}$ \\
\hline
\end{tabular}

Table 1. Interpretation of Equation 8 for Different Mean Flow Choices

input. While the $\partial q_{o} / \partial t$ term is generally non-zero in a time accurate simulation, the sum of $\partial q_{o} / \partial t$ with the fluxes must yield $Q_{o}=0$. It was initially assumed that this case would be as effective as using a converged solution for the mean where both $Q_{o}$ and $\partial q_{o} / \partial t$ are zero. In massively separated flow cases, one often cannot obtain a steady-state solution because of the inherent unsteadiness. In this event, another option for obtaining the NLDE mean input is to numerically compute the time-average of the time accurate simulation.

Table 1 summarizes the types of mean flow considered in this study, as well as a typical case involving flow with turbulence. The term "mean flow" is used interchangeably with mean input in this paper. Every mean input to the NLDE is not necessarily a true mean flowfield, the freestream case being an extreme example. In any case where $Q_{o}$ does not equal zero, the right-hand side of equation 4 acts as a source term for the unsteadiness. The instantaneous flowfield does not appear to be an acceptable choice. No physically correct NLDE solutions were obtained using this as a mean input. The exact reasons for this are still being investigated. The mean components are provided by any validated CFD code. Using a time-averaged mean requires more total (mean flow code plus NLDE code) computation time, but is included because of the possibility that the mean flow solver will not converge. It is also possible that the mean flow field may only be partially converged, such as the symmetric flowfield about a circular cylinder before unsteadiness begins. Both of these possibilities, as well as using a freestream flow as mean input, are addressed in this study.

The last row of table 1 represents a case not considered in this study. The converged Reynolds Averaged Navier-Stokes (RANS) case assumes a steadystate solution can be obtained for a flow involving both mean unsteadiness and unsteadiness due to turbulence. In contrast to the laminar counterpart in row 1 of a converged, steady solution, $Q_{o}$ does not equal zero for the converged RANS case. This is because the RANS equations include not only the terms in equation 8 , but also the Reynolds stresses, which are not accounted for in the definition of $Q_{o}$ given here.

\section{$\underline{\text { Numerical Details }}$}

\section{Spatial and Temporal Discretization}

The NLDE equations are discretized using the finite volume method of Jameson, Schmidt, and Turkel ${ }^{10}$. This classic method uses a blend of second and fourth order dissipation terms for stability. In the present work, the dissipation operator is applied in an analogous manner to the vector of conserved variables in equation 6 . The discretized domain in each test case uses an "O" grid 
composed of quadrilateral cells. A standard $4^{\text {th }}$ order Runge-Kutta integration is used to explicitly advance the solution in time.

\section{Boundary Conditions}

The boundary conditions by Tam and Dong ${ }^{11}$ are used for the farfield boundaries. Each cell on the boundary is evaluated as either an inflow or outflow boundary. For subsonic conditions, an inflow boundary may be classified as a radiation boundary, where the interior of the computational domain affects the boundary through pressure perturbations only. With a time invariant mean flow the radiation boundary conditions of Tam and Dong may be written as

$$
\left(\frac{1}{V(r, \theta)} \frac{\partial}{\partial t}+\frac{\partial}{\partial r}+\frac{1}{2 r}\right)\left[\begin{array}{l}
\rho^{\prime} \\
u^{\prime} \\
v^{\prime} \\
p^{\prime}
\end{array}\right]=0
$$

where $\mathrm{V}$ is defined by

$$
V(r, \theta)=\vec{V} \bullet \hat{e}_{r}+\left(a^{2}-\left(\vec{V} \bullet \hat{e}_{\theta}\right)^{2}\right)^{1 / 2}
$$

and $r$ is the distance from the boundary cell center to the origin of the coordinate system. In equation $11, a$ is the local speed of sound, $\vec{V}$ is the mean velocity vector, and $\boldsymbol{e}_{\mathrm{r}}$ and $\boldsymbol{e}_{\theta}$ are unit vectors in the radial and tangential directions, respectively. It is assumed that the origin of the coordinate system is in the center of the domain. Tam and Dong report the performance of these boundary conditions to be insensitive to the origin as long as the source of the perturbations is not located too close to the boundary. Equation 11 simply accounts for the speed of acoustic disturbances (propagation speed plus mean flow) along any $\theta$ direction.

Outflow boundaries may experience any combination of pressure, vorticity, or entropy disturbances. The numerical condition from Tam and Dong for pressure on these boundaries is

$$
\left(\frac{1}{V(r, \theta)} \frac{\partial}{\partial t}+\frac{\partial}{\partial r}+\frac{1}{2 r}\right) p^{\prime}=0
$$

and for density and velocity perturbations are

$$
\begin{aligned}
& \frac{\partial \rho^{\prime}}{\partial t}+\vec{V}_{o} \bullet \nabla\left(\rho^{\prime}\right)=\frac{1}{a_{o}^{2}}\left(\frac{\partial p^{\prime}}{\partial t}+\vec{V}_{o} \bullet \nabla\left(p^{\prime}\right)\right) \\
& \frac{\partial u^{\prime}}{\partial t}+\vec{V}_{o} \bullet \nabla\left(u^{\prime}\right)=-\frac{1}{\rho_{o}} \frac{\partial}{\partial x}\left(p^{\prime}\right) \\
& \frac{\partial v^{\prime}}{\partial t}+\vec{V}_{o} \bullet \nabla\left(v^{\prime}\right)=-\frac{1}{\rho_{o}} \frac{\partial}{\partial y}\left(p^{\prime}\right)
\end{aligned}
$$

Note that equation 12 is the same as the radiation condition for pressure in equation 10. Equations 10 - 13 are applied to each farfield boundary ghost cell at each time step. The boundary equations are discretized using a first order backward difference in time, and a second order backward difference for the spatial derivatives. The resulting finite difference formula is an explicit, algebraic expression for the perturbation value in the ghost cell. This ghost cell value is then used to calculate the appropriate values of the conserved variables in equation 6. Note that only the boundary condition for the perturbations is applied. The mean flow will automatically satisfy the boundary conditions.

The wall boundary conditions enforce zero velocity and zero pressure gradient at the wall. These conditions are applied only to the perturbations, because the mean flow already satisfies the wall boundary conditions.

\section{Mean Flow}

The mean flow is obtained from the baseline finite volume code (FLOW). A separate mean flow solution is not needed for the scattering test because the fluid is quiescent. The cylinder test cases all use the same grid for both the mean flow solution and the NLDE solution. In general, the grids need not be the same, and should not when very fine viscous grids are needed. The mean flow may be determined on a coarse grid using as little computation time as possible, and then interpolated to the finer mesh used in NLDE. Since none of the physics of the Navier-Stokes equations is removed or approximated in forming the NLDE equations, it was hypothesized that any flow field could be used as the mean and the NLDE perturbations should adjust to provide the correct total solution (mean plus perturbation). Using the freestream values of all flow variables as the mean flow field is tested to investigate this hypothesis. Since a uniform freestream obviously does not satisfy wall boundary conditions, a simple adjustment to their application is required. Instead of applying the wall conditions to just the perturbations, they are enforced on the total flow. The appropriate perturbations at the wall can then be calculated based on the identity that the total flow equals the freestream value plus the perturbation value. 


\section{$\underline{\text { Results and Discussion }}$}

\section{$\underline{\text { Scattering from a Circular Cylinder }}$}

The first test case is the scattering of a Gaussian pulse by a circular cylinder, selected from the $2^{\text {nd }}$ Computational Aeroacoustics Workshop on Benchmark Problems $^{12}$ (Problem 2, Category 1). The analytical solution is provided by Kurbatskii ${ }^{13}$. This case is chosen to evaluate the ability of the NLDE algorithm to accurately propagate waves, ensure both the farfield and wall boundary conditions are effectively implemented, and for direct comparison of the NLDE solution with the baseline finite volume code. The problem description from reference 12 is summarized here. Figure 1 diagrams the problem.

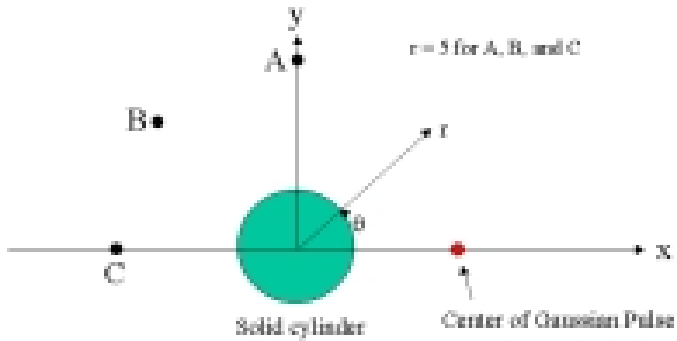

Figure 1. Scattering Benchmark Problem

All length references are dimensionless, based on cylinder diameter. The center of the two-dimensional Gaussian pulse is located four units from the cylinder center. The observer locations (points A, B, and C) are five units from the cylinder center as shown. The angular positions of points $\mathrm{A}, \mathrm{B}$, and $\mathrm{C}$ are at $\theta=90$, 145 , and 180 degrees, respectively. The initial spatial distribution of the perturbation pressure is given by

$$
p^{\prime}=\exp \left[-\ln 2\left(\frac{(x-4)^{2}+y^{2}}{0.2^{2}}\right)\right] \text {. }
$$

The maximum amplitude of the perturbation is one. There is zero mean flow for this problem. The grid used for this case is 1000 points in the circumferential direction and 600 points in the radial direction. The farfield boundary is placed eight diameters from the cylinder surface. A CFL number of one is used in the time stepping.

Figure 2 plots the pressure-time history (nondimensional) solutions for the benchmark scattering problem using the NLDE code. The FLOW code produced the same results as the NLDE code. The diminished amplitudes compared to the analytical response were anticipated since the baseline algorithm is only second order accurate. Figure 3 illustrates the effectiveness of the Tam and Dong boundary conditions at the farfield. The first frame is the pressure field after

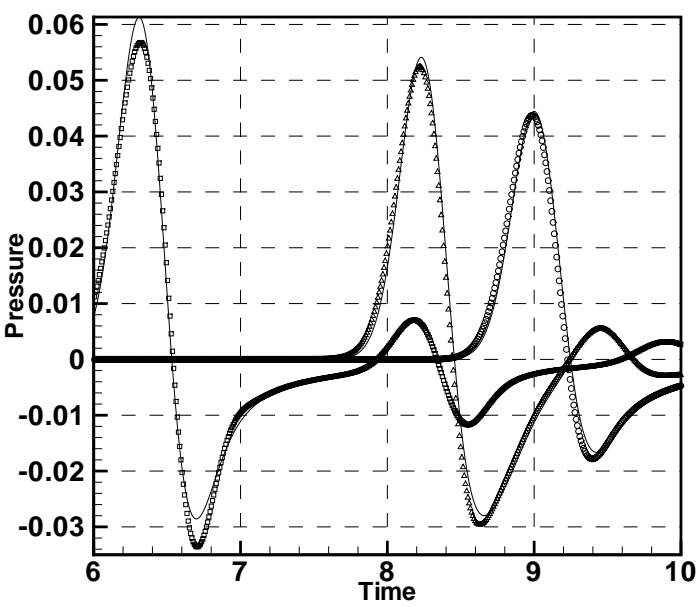

Figure 2. Scattering Problem Solution

(— Exact ; $\square \quad$ NLDE: Point A; $\triangle$ NLDE

Point B; O NLDE : Point C )

2000 time steps. The peak of the pulse has just reaches the cylinder surface and part of the exterior boundary. The non-dimensional time is 3.84. After 4000 time steps $(\mathrm{t}=7.64)$, the pulse has passes the cylinder, generating a scattered wave. The farfield boundary exhibits negligible reflections as the pulse exits the domain.

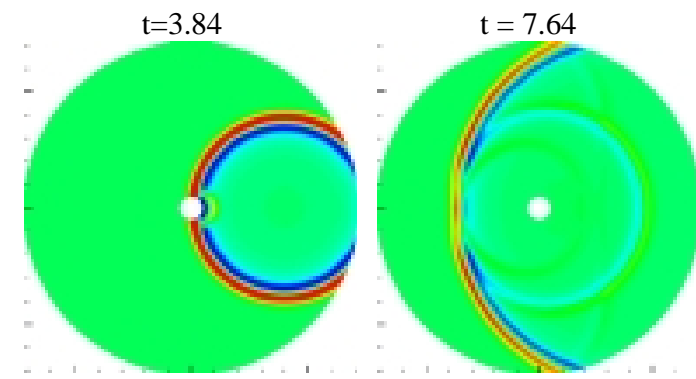

Figure 3. Gaussian Pulse Impinging on Farfield Boundary

\section{Circular Cylinder Flow}

The second series of tests involve flow past a circular cylinder. Of key interest is the accuracy of the unsteady vortex shedding behavior and the time required to generate an NLDE solution compared to obtaining the solution with the baseline finite volume code. An Ogrid using 301 equally spaced points in the circumferential direction and 65 points in the radial direction is used. The grid is stretched in the radial direction. The distance of the first cell center normal to the cylinder surface is $1 / 500$ of the cylinder diameter. The largest cell aspect ratio is approximately three. 
Conditions of Reynolds number 1000, and Mach number of 0.3 are used. While the boundary layer on the cylinder surface is still laminar at this Reynolds number, the wake has already transitioned to turbulence in the real, threedimensional, flow. Zero second order artificial viscosity is used. Where standard fourth order artificial viscosity is referred to in subsequent sections, the coefficient value of 1/256 originally recommended by Jameson et $a l .{ }^{10}$ is used. All computations are carried out on a single node of a PC cluster with a $400 \mathrm{Mhz}$ processor speed. Additional details of the PC cluster used are documented by Modi and Long ${ }^{14}$.

Using the standard value for fourth order artificial dissipation, initial tests of the cylinder demonstrated a resistance to the onset of periodic vortex shedding when a CFL number of one was used. Two methods were used to assist the initiation of unsteadiness. The first was the introduction of freestream turbulence at the inflow boundary. This method was not successful. Up to $15 \%$ freestream perturbations were used at the inflow boundaries with no impact toward initiating periodic vortex shedding. Breuer ${ }^{15}$ obtained similar results when introducing freestream turbulence for square cylinder computations. As Breuer states, this is due in part to the larger cells near the boundaries that tend to damp out the disturbances that are introduced. The disturbances do reach the cylinder, but cause lift perturbations only on the order of $10^{-5}$.

The second method was to introduce an asymmetric disturbance close to the cylinder. After the mean flow had sufficiently developed over the cylinder, the streamwise velocity in the upper half-plane is multiplied by 1.05 for one time step. This is similar to the approach by Hardin and Lamkin $^{16}$ who used a vorticity perturbation to initiate unsteadiness. In the case of the FLOW code, the cylinder forces begin responding immediately after this perturbation is introduced, slowly building to their periodic states. For the NLDE cases, the fourth order artificial viscosity coefficient was reduced to $20 \%$ of its standard value. This value was determined through trial and error. This value seems reasonable given that the mean flow can only generate disturbances through interactions with the perturbations, which are much smaller. This technique of "starting" the unsteadiness made it more difficult to assess the actual time needed to reach periodicity. Experimentation with higher CFL numbers, closer to the limits of stability for the fourth-order Runge-Kutta time integration, revealed that self-starting could be achieved without affecting the final solution. A CFL number of 2.7 is subsequently used in all cases reported below.

Results for the periodic state of vortex shedding are given in Table 2. These results are obtained over ten periods of shedding. The column headings indicate the code used. The NLDE cases differ by how the mean is obtained. NLDE1 uses a mean that is obtained from the
FLOW code by averaging over ten complete cycles of shedding. NLDE2 uses a mean obtained by pseudo-time marching the FLOW code until large, stable, recirculating zones were obtained in the cylinder wake. The NLDE3 case uses a freestream flowfield as the mean.

\begin{tabular}{|c|c|c|c|c|}
\cline { 2 - 5 } \multicolumn{1}{c|}{} & FLOW & $\begin{array}{c}\text { NLDE } \\
1\end{array}$ & $\begin{array}{c}\text { NLDE } \\
2\end{array}$ & $\begin{array}{c}\text { NLDE } \\
3\end{array}$ \\
\hline $\mathrm{C}_{1 \min }$ & -1.503 & -1.465 & -1.607 & -1.581 \\
\hline $\mathrm{C}_{l_{\max }}$ & 1.493 & 1.463 & 1.605 & 1.580 \\
\hline $\mathrm{C}_{l_{\text {avg }}}$ & -0.0077 & -0.0091 & -0.0025 & -0.0005 \\
\hline $\mathrm{C}_{\mathrm{d} \min }$ & 1.351 & 1.300 & 1.373 & 1.360 \\
\hline $\mathrm{C}_{\mathrm{d} \max }$ & 1.805 & 1.730 & 1.818 & 1.775 \\
\hline $\mathrm{C}_{\mathrm{d} \text { avg }}$ & 1.589 & 1.515 & 1.606 & 1.556 \\
\hline$\theta_{\mathrm{s}}$ & 94.6 & 95.0 & 90.6 & 95.8 \\
\hline $\mathrm{St}$ & 0.253 & 0.216 & 0.243 & 0.217 \\
\hline
\end{tabular}

Table 2. Time-Averaged Results for Circular Cylinder Test using FLOW code and NLDE code with Different Mean Inputs.

Figure 4 plots the streamlines for the mean flow obtained by time averaging. The lack of recirculating zones in the cylinder wake is typical of two-dimensional solutions where three-dimensional physics plays a role in the real flow. Breuer ${ }^{15}$ demonstrated this phenomenon when solving the flow over a circular cylinder using both a 2D and 3D simulation, both without turbulence modeling. Even when turbulence modeling is applied, a time-average solution similar to figure 4 can result due to weaknesses of the turbulence model. Such a case is discussed by Rodi ${ }^{17}$.

NLDE2 uses a mean obtained by pseudo-time marching the FLOW code with a CFL=1.0. The FLOW code was run until the convergence was down five orders of magnitude. Figure 5 shows the mean flow streamlines obtained using a pseudo-time marching solution. The mean flow for this case has $\mathrm{C}_{1}=-0.0050$, $\mathrm{C}_{\mathrm{d}}=0.717$, and $\theta_{\mathrm{s}}=83.9$. The same streamline values are used in both figures 4 and 5 .

The NLDE3 case uses freestream conditions as the mean flow. This requires the wall boundary conditions to be applied to the total solution since a freestream flow does not satisfy the wall boundary conditions. Farfield boundary conditions are satisfied by a freestream mean.

In table 2 , the angle of separation is $\theta_{\mathrm{s}}$ and is determined by the point where the time-averaged coefficient of friction becomes zero. The Strouhal 


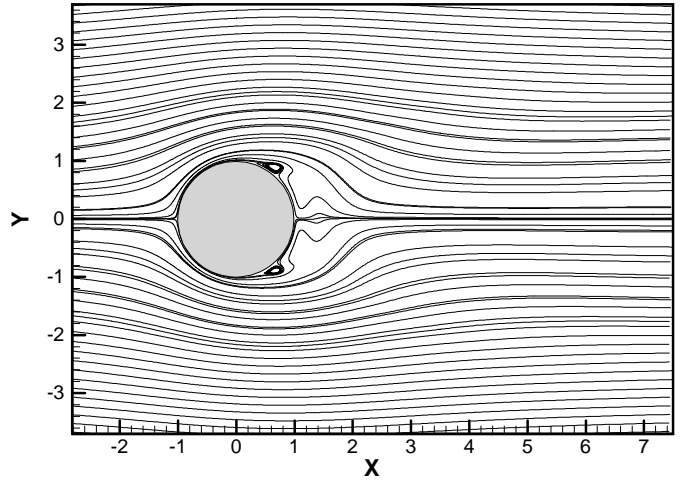

Figure 4. Time-averaged streamlines for the mean flow used in Case NLDE1.

number is based on the period of the lift coefficient cycle. The time step, required by stability considerations, is $6.1 \times 10^{-6}$. The generally accepted experimental values ${ }^{18}$ for $\mathrm{Re}=1000$ are Strouhal number $=0.20-0.22, C_{d}=1.0$, and $\theta_{\mathrm{s}}=80.5$. The drag coefficient was expected to be in error because of the relatively coarse discretization used next to the surface. Despite this, the data compares well with a numerical experiment conducted by Cox et al. ${ }^{19}$ The lift

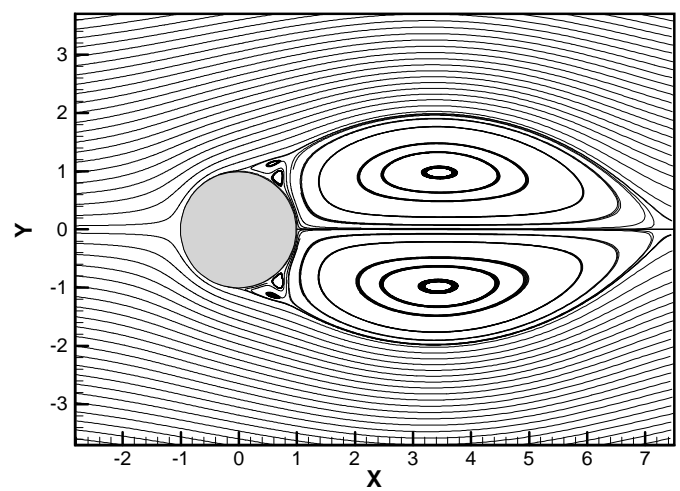

Figure 5. Streamlines for the mean flow obtained by pseudo-time marching used in Case NLDE2.

and drag extremes are approximately the same as reference 19 and the Strouhal prediction for the NLDE1 and NLDE3 cases are significantly better. Cox et al. ${ }^{19}$ used the code CFL3D and the distance to the first point off the cylinder surface was an order of magnitude smaller than the present study. The results shown here are, therefore, considered a valid basis for comparison between the baseline FLOW code and the NLDE version.

NLDE1 provides slightly improved drag estimates, a slightly less accurate separation angle, but a Strouhal number that is within the experimental range. NLDE2 provides drag estimates that are less accurate, a mean lift that is closer to zero, a separation angle that is very accurate, and a Strouhal number that is only marginally better than the FLOW code. NLDE3 provides improved estimates in each of the measured parameters, with the improvement in Strouhal number being the most significant.

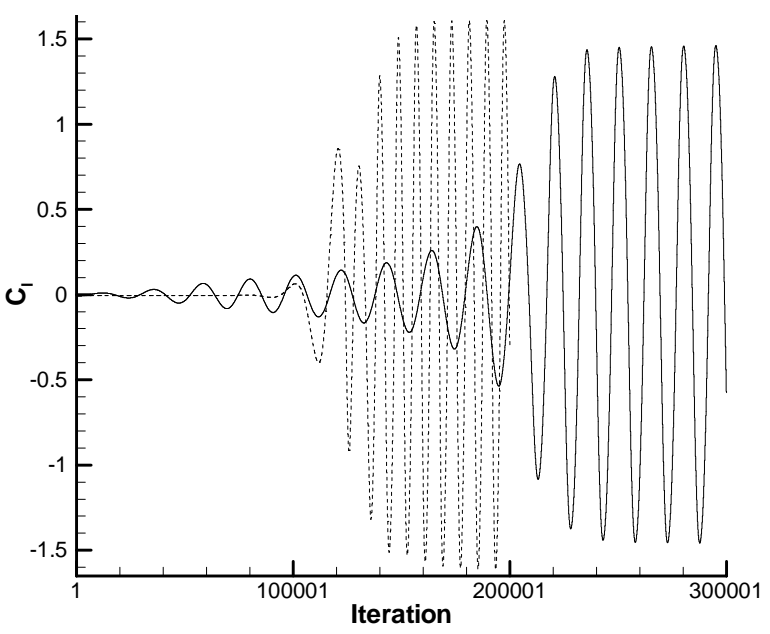

Figure 6. Lift Coefficient History for NLDE1 and NLDE2 ( - NLDE1; - - - NLDE2 )

NLDE1 represents the case where convergence is unattainable, so a time average is used to represent the mean. In this event, the $Q_{o}$ term behaves as a source term and must be retained in the equation set. If a well converged mean solution can be obtained as in NLDE2, time averaging is not required and $Q_{o}=0$. Figure 6 plots the lift coefficient history for these two cases. The NLDE2 converges to a periodic state in fewer iterations, but as already noted is not as accurate in either drag coefficient or frequency of shedding. NLDE1 begins unsteadiness from the start of the NLDE solution, but does so slowly over about 240,000 iterations before reaching a periodic state.

The NLDE3 case uses the same mean as the initial condition in the FLOW code, and a direct comparison of the number of cycles needed to obtain periodicity can be made. Comparisons of lift and drag coefficient convergence are shown in figures 7 and 8 . The variable "vis4" in the legend refers to the fraction of standard fourth order artificial viscosity used. The NLDE3 run using vis $4=0.2$ is observed to converge to the periodic state much more quickly than the FLOW case using the standard fourth order viscosity (vis4 = 1.0). To make sure this is not a damping effect of artificial viscosity, the FLOW code was also run at $60 \%$ and $40 \%$ of the standard artificial dissipation. The FLOW code becomes unstable when $20 \%$ viscosity is attempted. The comparisons in figures 7 and 8 show that convergence to the periodic state is delayed in the FLOW code when smaller values of artificial viscosity are used. This result was unexpected. Once the periodicity does begin for the lower values of artificial viscosity, the peaks are much 7 
more erratic, which is expected because of the smaller

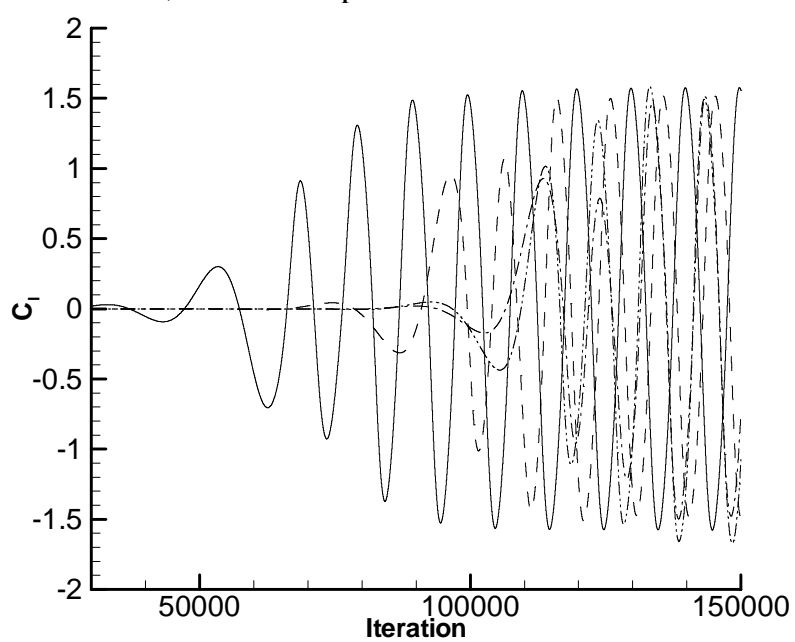

Figure 7. Lift Coefficient History Comparison

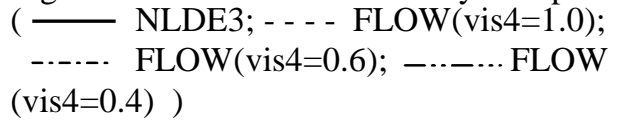

damping. The NLDE3 case obtains a fully periodic state by iteration 105,000 while the peaks of all the FLOW cases still fluctuate past 150,000 iterations. The FLOW case using standard viscosity reaches consistent periodicity at iteration 180,000 , while the $60 \%$ and $40 \%$ cases fail to converge to a periodic state even at 200,000 iterations when the calculation is terminated.

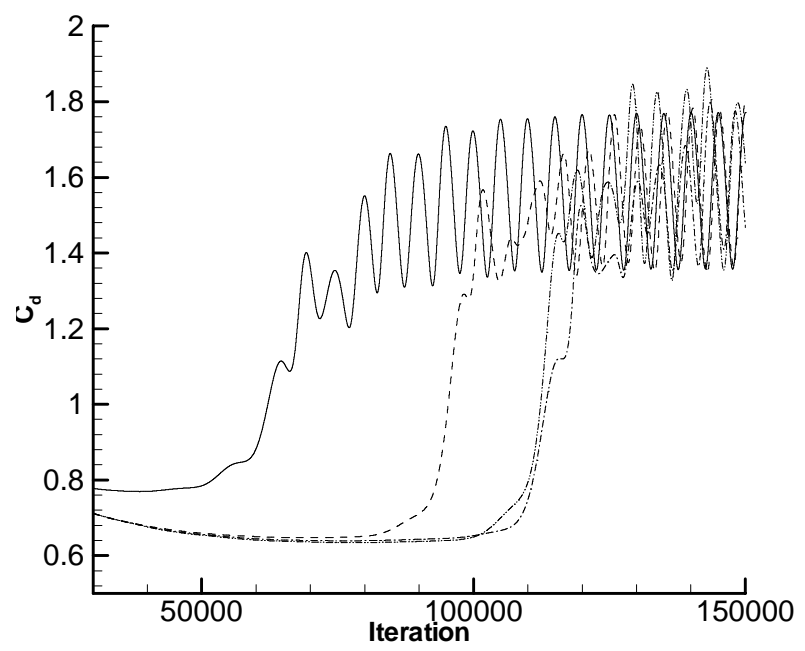

Figure 8. Drag Coefficient History Comparison

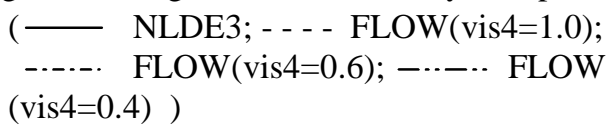

Figure 9 is a contour plot of the absolute values of vorticity near the cylinder obtained from an NLDE3 solution. The shed vortex detaches between two and three cylinder diameters downstream of the cylinder surface. The vorticity structure is very complex near the cylinder surface.
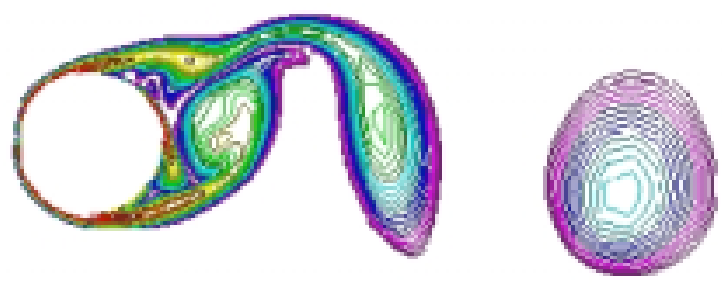

Figure 9. Absolute Vorticity Contours During Periodic Shedding.

Table 3 summarizes the differences between the various mean flow inputs and the corresponding NLDE solution as a percentage error relative to the experimental values. In the case of the freestream (NLDE3), the average $\mathrm{C}_{\mathrm{d}}$ data is listed as not applicable (NA) because the freestream mean does not satisfy the cylinder wall boundary conditions. Positive numbers in the drag, separation angle, and Strouhal number rows indicate the numerical value is above the experimental value. Negative numbers indicate the numerical value is below the experimental value. The NLDE1 case has the strongest correlation between accuracy of the mean flow and the accuracy of the corresponding NLDE solution. The time-averaged mean used in NLDE1 contains more information about the flow than the other two cases. The NLDE2 mean contains no information about the frequency of unsteadiness, so NLDE1 is expected to yield a more accurate Strouhal number. The NLDE2 mean contains a much more accurate separation angle than the other cases, and provides the best NLDE solution for this parameter. The NLDE3 case appears to violate the hypothesis that the more accurate the mean, the more accurate the NLDE solution. The NLDE3 mean contains no information about the true mean flowfield, yet the NLDE solution gives a Strouhal number within the spread of experimental data, a drag prediction that is better than NLDE2, a separation angle almost as good as NLDE1, and the most accurate lift solution. This suggests that providing a mean flow with "neutral" information as in NLDE3 may be better than using a mean flow that contains inaccurate information.

\section{Conclusions}

The first test of the NLDE algorithm that includes the effects of viscosity with a wall-bounded flow has been conducted here. These tests are also the first effort to include the viscous perturbation terms in any NLDE simulation. The results are specific to the test cases explained and may not be generalized until further testing is completed. For the unsteady, inviscid test case, 8 


\begin{tabular}{|l|c|c|c|c|c|c|c|}
\cline { 3 - 8 } \multicolumn{2}{c|}{} & \multicolumn{2}{c|}{ NLDE1 } & \multicolumn{2}{c|}{ NLDE2 } & \multicolumn{2}{c|}{ NLDE3 } \\
\cline { 2 - 8 } \multicolumn{1}{c|}{} & Exp. & $\begin{array}{c}\text { Mean } \\
\text { Input }\end{array}$ & $\begin{array}{c}\text { NLDE } \\
\text { Solution }\end{array}$ & $\begin{array}{c}\text { Mean } \\
\text { Input }\end{array}$ & $\begin{array}{c}\text { NLDE } \\
\text { Solution }\end{array}$ & $\begin{array}{c}\text { Mean } \\
\text { Input }\end{array}$ & $\begin{array}{c}\text { NLDE } \\
\text { Solution }\end{array}$ \\
\hline $\mathbf{C}_{\mathbf{d} \text { avg }}$ & 1.0 & +58.9 & +51.5 & -28.3 & +60.6 & NA & +55.6 \\
\hline $\boldsymbol{\theta}_{\mathbf{s}}$ & 80.5 & +17.5 & +18.0 & +4.2 & +12.5 & -100 & +19.0 \\
\hline $\mathbf{S t}$ & $0.21-$ & +15.0 & +0.5 & -100 & +13.0 & -100 & +0.9 \\
\hline
\end{tabular}

Table 3. Percent Differences Relative to Experimental Values

no advantages of the NLDE algorithm are discernable over the baseline code. For the unsteady, viscous case the value of fourth order viscosity is found to be an important factor in obtaining the onset of periodic shedding as well as achieving a converged periodic state. The NLDE were found to operate suitably using only $20 \%$ of the standard fourth order artificial dissipation. The use of three different types of mean flow were studied as inputs to the NLDE formulation. Mixed results were obtained when using either a time-averaged or a pseudo-time marched steady-state solution for the mean flow. The benefits of increased accuracy obtained in one parameter were generally offset by reduced accuracy in one or more other parameters. Using a mean equal to the freestream provides the most intriguing results, providing good accuracy in all measured parameters, as well as converging to a periodic state using only $58 \%$ of the iterations needed for the FLOW code.

\section{$\underline{\text { References }}$}

${ }^{1}$ Morris, P.J., Long, L.N., Bangalore, A., Wang, Q., “A Parallel Three-Dimensional Computational Aeroacoustics Method Using Nonlinear Disturbance Equations", J. of Computational Physics, Vol. 133, 1997.

${ }^{2}$ Morris, P.J., Long, L.N., Lockard, D.P., Wang, Q., "Numerical Prediction of High Speed Jet Noise", AIAA Paper 97-1598, 1997.

${ }^{3}$ Morris, P.J., Long, L.N., Scheidegger, T.E., Wang, Q., Pilon, A.R., "High Speed Jet Noise Simulations", AIAA Paper 98-2290, 1998.

${ }^{4}$ Liu, J., Long, L.N., Modi, A., “ Higher Order Accurate Solutions of Ship Airwake Flow Fields using Parallel Computers", AGARD Conference, Amsterdam, 1998.
${ }^{5}$ Liu, J., Long, L.N., “ Direct Aeroacoustic and Aerodynamic Simulation of Multi-Hole Engine Liners", AIAA Paper 98-2330, 1998.

${ }^{6}$ Scheidegger, T.E., Morris, P.J., , and Long, L.N., "Jet Noise Simulations for Circular and Rectangular Nozzles", AIAA Paper 2000-2080, 2000.

${ }^{7}$ Chyczewski, T, Morris, P.J., and Long, L.N., "LargeEddy Simulation of Wall Bounded Shear Flow Using the NLDE”, AIAA Paper 2000-2007, 2000.

${ }^{8}$ Long, L.N., “A Non-Conservative Nonlinear Flowfield Splitting Method”, AIAA Paper 2000-1998, 2000.

${ }^{9}$ Sezer-Uzol, N. and Long, L.N., "High Accuracy Wake and Vortex Simulation Using a Hybrid CFD/DV Method", AIAA Paper 2000-2029, 2000.

${ }^{10}$ Jameson, A., Schmidt, W., and Turkel, E., "Numerical Solution of the Euler Equations by Finite Volume Methods Using Runge-Kutta Time Stepping Schemes", AIAA Paper $81-1259,14^{\text {th }}$ Fluid and Plasma Dynamics

Conference, 1981.

${ }^{11}$ Tam, C. K. W., and Dong, Z., "Radiation and Outflow Boundary Conditions for Direct Computation of Acoustic and Flow Disturbances in a Nonuniform Mean Flow", CEAS/AIAA Paper 95-007,1995.

${ }^{12}$ NASA Conference Publication 3352, $2^{\text {nd }}$ Computational Aeroacoustics Workshop on Benchmark Problems, Langley Research Center, Hampton, VA, June 1997.

${ }^{13}$ Kurbatskii, K., "Analytical Solutions of the Category 1, Benchmark Problems 1 and 2", NASA Conference Publication 3352, $2^{\text {nd }}$ Computational Aeroacoustics Workshop on Benchmark Problems, Langley Research Center, Hampton, VA, June 1997. 
${ }^{14}$ Modi, A. and Long, L.N., "Unsteady Separated Flow Simulations Using a Cluster of Workstations", AIAA Paper 2000-0272, 2000.

${ }^{15}$ Breuer, M., "Large Eddy Simulation of the Sub-Critical Flow Past a Circular Cylinder: Numerical and Modeling Aspects", International Journal for Numerical Methods in Fluids, Vol. 28, No., Dec. 1998, pp. 1281-1302.

${ }^{16}$ Hardin, J. and Lamkin, S., "Aeroacoustic Computation of Cylinder Wake Flow", AIAA Journal, Vol. 22, No. 1, January 1984.

${ }^{17}$ Rodi, W. "On the Simulation of Turbulent Flow Past Bluff Bodies", Proceedings of the $1^{\text {st }}$ International Symposium on Computational Wind Engineering, August 1992.

${ }^{18}$ Zdravkovich, M.M., Flow Around Circular Cylinders, Oxford University Press, Oxford, 1997.

${ }^{19}$ Cox, J. Rumsey, C., Brentner, K., and Younis, B. "Computation of Sound Generated by Viscous Flow Over a Circular Cylinder", NASA TM 110339,

March, 1999. 